\title{
Why NMDA-receptor-dependent long-term potentiation may not be a mechanism of learning and memory: Reappraisal of the NMDA-receptor blockade strategy
}

\author{
JULIAN R. KEITH and JERRY W. RUDY \\ University of Colorado, Boulder, Colorado
}

\begin{abstract}
The hypothesis that the processes mediating the induction of long-term potentiation (LTP) in the hippocampus also subserve hippocampally mediated learning and memory has recently been examined using an NMDA-receptor antagonist (AP5) to block the induction of LTP during training on learning tasks (Morris, Anderson, Lynch, \& Baudry, 1986; Staubli, Thibault, DiLorenzo, \& Lynch, 1989). The authors claim that their results support the NMDA-receptor-dependent LTP/memory hypothesis. Our reappraisal of the empirical support for this claim, however, suggests that it is premature, and that some aspects of the data can be used to argue just the opposite: that NMDA-receptor-dependent synaptic changes are not critically involved in the learning and memory processes that were under investigation. We support our position by arguing that (1) these studies did not convincingly rule out sensorimotor interpretations of the behavioral impairments that were observed, and (2) in all tasks studied, the AP5-treated animals displayed substantial evidence of learning, a result that contradicts the NMDA-receptor-dependent LTP/memory hypothesis. We also consider data from studies in which the noncompetitive NMDA antagonist MK-801 was used, and we conclude that the results obtained with this agent cannot be used to support the LTP/memory hypothesis, because MK-801 does not block NMDAreceptor-dependent LTP in vivo in doses that permit animals to behave.
\end{abstract}

It is generally accepted that learned changes in behavior depend on experience-induced alterations in synaptic connections among neurons. The task of translating this general concept into specific neural mechanisms has engaged many researchers, and it is one of the central challenges that face behavioral neuroscientists.

The discovery of long-term potentiation (LTP) (Bliss \& Gardner-Medwin, 1973; Bliss \& Lomo, 1973) is regarded by many as an important step in the advancement of our understanding of the neuronal basis of learning and memory. Specifically, Bliss and his colleagues discovered that a volley of electrical impulses applied to perforant path fibers that synapse on the granule cells of the dentate gyrus can give rise to synaptic changes that may persist for weeks. Since the discovery of LTP in the hippocampus, many other areas of the brain have been found to be susceptible to LTP (see Teyler \& DiScenna, 1987, for a review), and LTP has become a prime memory storage candidate.

The authors thank Maria Alvarado, Robert Eaton, Jeff Galef, Eugene Glass, Peter Kaplan, Steve Maier, and Robert Sutherland for their thoughtful comments on previous versions of this manuscript. Correspondence should be addressed to Julian R. Keith or Jerry W. Rudy, Campus Box 345, Department of Psychology, University of Colorado, Boulder, CO 80309 .
The critical issue concerning LTP, however, was articulated by Bliss and Lomo (1973) in their original report:

Whether or not the intact animal makes use in real life of a property which has been revealed by synchronous repetitive volleys to a population of fibers the normal patterns of activity along which are unknown, is another matter. (p. 355)

Thus, a fundamental challenge to behavioral neuroscientists is to provide a plausible connection between this artificially induced change in synaptic strengths and memorybased behavior.

A recent advance in the understanding of the processes necessary for the induction of LTP now permits the relationship between LTP and memory to be approached from a new direction. Specifically, the induction of some forms of LTP is dependent on the activation of $N$-methyl-Daspartate (NMDA) receptors. The induction of this form of LTP in the hippocampus can be prevented by the administration of an NMDA receptor antagonist, such as D-amino-phosphono-valeric acid (AP5) (Collingridge, Kehl, \& McLennan, 1983; Harris, Ganong, \& Cotman, 1984; Morris, Anderson, Lynch, \& Baudry, 1986).

NMDA receptor antagonists thus provide a relatively direct way of relating NMDA-receptor-dependent synaptic changes to memory-based behaviors. The logic of the argument is simple (Brown, Chapman, Kairiss, \& Keenan, 
1988; Morris et al., 1986; Staubli, Thibault, DiLorenzo, \& Lynch, 1989). If some forms of learning and memory are mediated by NMDA-receptor-dependent processes, then blocking the induction of NMDA-receptor-dependent LTP should prevent the acquisition of memory-based performance that depends on such processes. According to this argument, the hypothesis that some forms of learning and memory are subserved by the neural mechanisms that mediate NMDA-receptor-dependent LTP is supported if animals treated with an NMDA antagonist fail to learn.

A cursory review of the literature on research employing the NMDA blockade strategy would suggest that the hypothesis has met with some empirical support. For example, consider these three conclusions taken from the abstracts of papers by Morris et al. (1986), Staubli et al. (1989), and Robinson, Crooks, Shinkman, and Gallagher (1989):

These results suggest that NMDA receptors are involved in spatial learning, and add support to the hypothesis that LTP is involved in some, but not all, forms of learning. (Morris et al., 1986, p. 774)

We propose that the results are consistent with the hypothesis that NMDA receptors are involved in those forms of learning that involve modification of connections in the forebrain. (Staubli et al., 1989, p. 54)

These results are consistent with the view that an NMDA mechanism is critical for certain forms of learning. (Robinson et al., 1989, p. 156)

The conclusion that AP5 impairs learning has also made its way into a recent review paper that appeared in Science, where Brown et al. (1988), in reference to the Morris et al. (1986) paper, wrote: "One study that was designed to explore this possibility demonstrated that intraventricular administration of AP5 prevents learning a new spatial memory task" (p. 727).

That a plausible linkage between NMDA-receptordependent LTP and memory-dependent performance may have been established is both exciting and important. It would mean that what has been learned about the anatomy, physiology, and biochemistry underlying artificially induced LTP would be directly applicable to our understanding of learning and memory per se. In view of these implications, it is particularly appropriate at this early stage of investigation to critically evaluate the experimental basis from which the conclusion above has been derived and to consider alternative interpretations of these data. Ironically, our review has led us to conclude that some aspects of the data can be used to argue against a role for NMDA-receptor-dependent synaptic changes in learning and memory.

\section{GENERAL INTERPRETATIONAL ISSUES}

Before presenting our analysis of studies in which the NMDA-receptor blockade strategy has been employed, we will consider the general issue of the kind of evidence that can be interpreted as supporting the hypothesis that NMDA-receptor-dependent LTP is a plausible storage mechanism for some forms of memory. Within this framework, the minimally acceptable starting point for supporting this hypothesis is only met when an NMDA-receptor antagonist (1) blocks the induction of LTP in at least one set of synapses of a circuit known to be critical to some learned behavior change, and (2) impairs performance on a learning task known to depend on that circuit. Once this preliminary test has been met, the researcher must then address the problems raised by the learning-versusperformance distinction. The problem is that impaired performance on a learning task may be due to the effect of the antagonist on sensory, motor, or motivational processes that must function normally for the animal to perform appropriately on the task, and not due to its effect on learning and memory processes. Unless such nonassociative interpretations of the behavioral impairment can be dismissed, the claim that the agent impaired learning and memory is unjustified. Even under the best circumstances (i.e., when the agent both interferes with the induction of LTP and impairs performance, and when nenassociative effects can be excluded), the researcher can only conclude that the results are consistent with the hypothesis. This approach cannot in principle provide direct support for the hypothesis that the induction of LTP is a necessary condition for learning and memory.

What has gone unappreciated in that the NMDAreceptor blockade approach, in principle, can lead to an outcome that permits a much stronger conclusion. Specifically, there is a set of outcomes that would permit one to reject the hypothesis that NMDA-receptor-dependent LTP is a memory mechanism. This conclusion would be appropriate if the agent blocked the induction of LTP in a neural circuit known to be critical for some learned behaviors, and if animals treated with the agent clearly displayed the ability to acquire those behaviors.

In the remainder of this paper, we will defend our position that the weight of the evidence favors the rejection of NMDA-receptor-dependent LTP as a plausible memory storage candidate. We will argue that (1) the evidence indicates that animals treated with AP5 display substantial learning, even when trained on tasks considered to be the ones most likely to be impaired by an NMDAreceptor blockade, and (2) the extent to which the performance of AP5-treated animals is impaired can be plausibly attributed to the nonassociative effects of AP5. Even if the first of these points should be disputed, the second one will provide sufficient grounds for dismissing previous claims that NMDA-receptor-dependent synaptic alterations are necessary for some forms of learning.

We will focus this commentary primarily on results reported in two recent papers, one by Morris et al. (1986), and the other by Staubli et al. (1989). In both studies, tasks were used that are sensitive to interference with the nor- 
mal functioning of the hippocampus, and AP5 was administered in a way that blocks the induction of LTP in the dentate area of the hippocampus in vivo. We have no reason to dispute the conclusion that the induction of LTP was prevented in the animals whose behavior was tested. ${ }^{1}$ We challenge, however, the authors' conclusion that AP5 interfered with learning, and we contend that the most important aspect of both studies is that, in spite of being treated with AP5, animals displayed substantial learning in all tasks studied. We will then consider recent findings obtained with another NMDA-receptor antagonist, MK-801, and conclude that they are, at best, irrelevant to the issue under debate, because MK-801 does not block the induction of LTP, in vivo in doses that permit the animal to behave.

\section{The Morris, Anderson, Lynch, and Baudry (1986) Study}

Morris et al. (1986) examined the effects of blocking NMDA-receptor-dependent LTP on the acquisition of both a place learning task and a nonspatial visual discrimination problem. These authors concluded that APS had no effect on visual discrimination learning but that it impaired performance on a hippocampally mediated place learning task. In the place learning task, the intertrial interval (ITI) was $4 \mathrm{~h}$, and the animals had to learn the location of an escape platform that was hidden below the water surface in a circular swimming pool. AP5-treated rats were impaired (i.e., they exhibited longer escape latencies) relative to control animals during early stages of training. Critical evidence that AP5-treated animals did not learn the location of the platform, however, was derived from a 60 -sec probe trial, in which the animals were tested with the platform removed from the pool. During the probe trial, the control animals demonstrated a bias for swimming in the quadrant in which the platform was located during training, a finding that is generally accepted as evidence of place learning. In contrast, the AP5-treated animals displayed no such bias. These results suggest that AP5 blocked place learning.

What needs to be considered, however, is that following the probe trial, while still under the NMDA blockade, the animals received an additional eight training trials with an ITI of $30 \mathrm{sec}$. Twenty-four hours after this retraining, Morris et al. (1986) tested the animals by reversing the location of the platform (i.e., the platform was placed diagonally opposite to its location during original training). The "reversal" procedure is a test of place learning (Sutherland \& Dyck, 1984).

The rationale for the reversal test as a measure of place learning assumes that an animal that learned the spatial location of the platform will initially search that location before searching other sites. This search pattern would result in relatively long escape latencies on reversal trials, relative to the original terminal acquisition latencies. In contrast, the escape latencies during reversal training of an animal that did not know the spatial location of the platform but was using a nonspatial strategy should not differ from its terminal acquisition latencies.

Animals with hippocampal damage, for example, behave on a reversal test as one would expect of animals incapable of place learning: Their latencies to escape on reversal trials do not differ from their latencies during original training (Sutherland, Whishaw, \& Kolb, 1983). The AP5-treated animals in the Morris et al. (1986) study, however, behaved as if they had learned the original spatial location of the platform. Their escape latencies increased from approximately $20 \mathrm{sec}$ on the final trial block when the platform was in its original location to approximately $60 \mathrm{sec}$ on reversal trials (see Morris et al., 1986, Figure 1). This result is strong evidence that AP5-treated animals did learn the original location of the platform.

What significance, then, should be attached to the findings that these animals (1) displayed longer escape latencies than the control animals did over the initial 15 training trials, and (2) failed to display a quadrant bias on the probe trial? Our view is that these findings reflect the nonassociative sensorimotor and motivational effects of the drug. It is known that AP5 can produce sensorimotor impairments. As has been noted by Mondadori, Weiskrantz, Buerki, Petschke, \& Fagg (1989), NMDA-receptor antagonists disrupt normal functioning of sensorimotor pathways (Cahusac, Evans, Hill, Rodriguez, \& Smith, 1984; Harris et al., 1982; Headley, Parsons, \& West, 1987; Polc, 1985; Salt, 1986), elicit muscular relaxation (Turski et al., 1985), and share some properties of the psychotomimetic drug phencyclidine (PCP) (Compton, Kreiter, Smith, Harken, \& Monahan, 1988; Koeck, Woods, \& Ornstein, 1986; Tricklebank, Singh, Oles, Wong, \& Iversen, 1987).

Additionally, AP5 blocks the atropine-sensitive hippocampal theta rhythm in behaving rats, presumably because of its effects on the septal area (Leung \& Desborough, 1988). As has been noted by Leung and Desborough (1988), the effects of AP5 observed by Morris et al. (1986) on performance during training on a place learning task could be due to a disruption of the theta rhythm or the normal sensorimotor processing associated with the theta rhythm. Leung and Desborough (1988) also reported that a decrease in locomotor activity usually accompanied AP5 administration.

Indeed, Morris et al. (1986) acknowledged the presence of sensorimotor impairments in their experiment: "Moreover, some D,L-AP5 animals occasionally fell off the escape platform on the first 2-3 days of training" (p. 774). Thus, it is not surprising that the escape latencies of AP5treated subjects were initially long in comparison with those of control animals, or that they failed to display a quadrant bias on the probe trial.

Morris et al. (1986) attempted to control for the sensorimotor impairments produced by AP5 by training animals on a visual discrimination task in the same apparatus. This task was chosen because successful performance on it does 
not require a normally functioning hippocampus. The performance of AP5-treated animals did not differ from that of control animals on the visual discrimination task. Mean trials to criterion were 76.8 and 80.6 , respectively, for the AP5-treated and control animals. This finding led Morris et al. to the conclusion that the impaired performance of AP5-treated animals on the place task was not the result of a sensorimotor impairment.

This conclusion, however, can be challenged on the grounds that Morris et al.'s (1986) control experiment was faulty in at least one crucial respect. What must be noted is that the ITI during the first phase of the place learning experiment was $4 \mathrm{~h}$, whereas the interval was $30 \mathrm{sec}$ during the visual discrimination experiment. This confounding of the ITI with the training task obviously precludes Morris et al.'s conclusion that the AP5-treated animals' impairment on the place learning task was not due to the sensorimotor and motivational disruptions produced by AP5. In this context, then, it is instructive to reconsider the fact that it was after Morris et al. trained their AP5treated animals on the place task with a $30-\mathrm{sec}$ ITI that the reversal test revealed that they had learned the location of the platform. It may be that the magnitude of the sensorimotor and motivational effects of NMDA-receptor antagonists depend on the ITI's being more robust when that interval is $4 \mathrm{~h}$ than when it is $30 \mathrm{sec}$.

Regardless of why AP5-treated animals were impaired on the place task when the ITI was $4 \mathrm{~h}$, it is clear that when the ITI was $30 \mathrm{sec}$, AP5-treated animals performed as well as controls on both the place learning task and the visual discrimination task. The important point to appreciate is that AP5 should block the induction of LTP, regardless of whether the ITI was $4 \mathrm{~h}$ or $30 \mathrm{sec}$. Performance on the place learning task, however, was disrupted only when a 4-h ITI was used. In light of the dissociation between the blockade of LTP and the preserved place learning abilities demonstrated by AP5-treated animals when the ITI was $30 \mathrm{sec}$, we consider it doubtful that the induction of LTP is the crucial factor in the determination of how animals performed on the place learning task.

Finally, it has been noted that the difficulties involved in interpreting the effects of AP5 on place learning performance are compounded by the fact that NMDA-receptor antagonists are known to have anxiolytic properties (Bennet \& Amrich, 1986; Clineschmidt, Martin, \& Bunting, 1982). This observation is important, because chlordiazepoxide, an anxiolytic benzodiazepine, produces virtually the same behavioral effects on place learning in the swimming pool as AP5 does (McNaughton \& Morris, 1987). Thus there is no basis for concluding that AP5 impairs place learning performance because it blocks NMDAreceptor-dependent LTP. It is equally plausible to conclude that the performance impairments that accompany the AP5 treatment are due to its anxiolytic properties.

Even though Morris et al. (1986) could have controlled more carefully for the nonassociative effects of AP5, ${ }^{2}$ one should not be distracted from the central finding of their study that AP5-treated animals displayed place learning.
During original training, these animals' escape latencies decreased from approximately 110 to $20 \mathrm{sec}$, and they were not different from the escape latencies of the control animals on the last trial block immediately prior to reversal training. Moreover, in our opinion, the reversal test trials revealed that AP5-treated animals learned the original location of the platform. Thus, AP5 did not impair place learning processes, even though it did block the induction of NMDA-dependent LTP.

\section{The Staubli, Thibault, DiLorenzo, and Lynch (1989) Study}

Staubli et al. (1989) examined the effects of AP5 on the performance of rats on two tasks. One involved the acquisition and retention of simultaneous olfactory discriminations; the other was a one-way active avoidance task. These tasks were chosen because both have been shown to be sensitive to hippocampal damage. We do not challenge Staubli et al.'s claim that AP5 did not impair avoidance learning. Indeed, AP5 facilitated avoidance learning. The results of the olfactory discrimination experiments, however, were interpreted as providing support for the LTP/memory hypothesis. We strongly challenge this conclusion.

Staubli et al. (1989) assessed the effects of AP5 an olfactory discrimination learning under three conditions that depended on the concentration of the odors and the ITI. These conditions comprised (1) normal odor intensity and a 10-min ITI, (2) low odor intensity and a 10-min ITI, and (3) low odor intensity and a 2-min ITI. First, it should be noted that AP5-treated animals did not differ from the control animals when trained under either Condition 1 or Condition 3. Differences between AP5-treated animals and controls only appeared when the odor concentrations were low and the ITI was $10 \mathrm{~min}$. Even in this condition, the AP5-treated animals readily solved the discrimination task, and they only differed from controls during early training. It is clear from Figure 2 of the Staubli et al. report that the only difference between the AP5-treated and the control animals was that the control animals gained a small advantage during the initial 5 training trials that disappeared after only 15 trials. This initial small advantage could easily have been the result of sensorimotor impairments produced by AP5.

Staubli et al. (1989) attempted to control for the sensorimotor impairments produced by AP5 by training animals on discrimination problems (using only normal odor concentrations) prior to AP5 treatment and assessing retention of these problems during administration of AP5. AP5 did not impair retention of the discrimination problems acquired prior to AP5 treatment. Staubli et al. suggested that this finding argues against a sensorimotor impairment interpretation of their data. It is important to note, however, that odor discrimination learning was never impaired by AP5 in the Staubli et al. study when normal odor concentrations were used. Thus Staubli et al.'s control for sensorimotor impairments is uninformative, because the concentration of the odors was confounded with whether 
retention (normal concentration) of a previously acquired problem or the acquisition (low concentration) of a new problem was being assessed.

It also should be noted that a lower dose of AP5 than the dose used by Staubli et al. (1989) has been found to alter the neural activity underlying normal respiration in the rat (McCrimmon, Smith, \& Feldman, 1989). This observation might explain the minor effect Staubli et al. (1989) obtained when the odor concentrations were low. Perhaps AP5 impaired the rats' ability to sample lowintensity olfactory stimuli, thus delaying conditioning during the early phases of training.

Two other aspects of the Staubli et al. (1989) data support the hypothesis that the minor difference that was reported was not a result of a learning impairment. First, after the 20 training trials on one odor discrimination problem (low concentration and 10-min ITI), Staubli et al. reversed the significance of the two odors (the previously correct odor was now the incorrect choice). Performance during reversal training can be considered a measure of the degree of original learning (see Mackintosh, 1974, pp. 601-610). If one animal learned the original problem better than another, that animal should have made more errors during reversal training than the animal that had not learned the original problem as well. Thus if AP5 significantly impaired the learning of the original discrimination, one would expect the control animals to have made more errors during reversal learning than did animals that were treated with AP5. The two sets of animals, however, did not differ at any point during reversal learning (see Staubli et al., 1989, Figure 3).

Second, another way of determining the strength of original learning is to assess the retention of a previously learned discrimination. If one animal learned the problem better than the other, one might expect that it would also retain that discrimination better. If AP5 interfered with learning, then an animal might display a retention impairment. After AP5 was presumably no longer present in the organisms, Staubli et al. (1989) tested the animals for retention of a previously learned odor discrimination (low concentration and 10-min ITI) and found no difference between the AP5-treated and control animals (see Staubli et al., Figure 5). The results of both the reversal and retention tests favor the hypothesis that the slight behavioral impairment Staubli et al. reported was not due to its effect on the learning process per se.

Thus the most impressive finding in the Staubli et al. (1989) study is how well the animals learned in all tasks while being trained under conditions that block the induction of NMDA-dependent LTP.

\section{Experiments Involving MK-801}

Robinson et al. (1989) based their claim that an "NMDA mechanism is critical for certain forms of learning" (p. 156) on their findings that pretrial injections of the noncompetitive NMDA antagonist (+)-5-methyl-10,11dihydro-5H-dibenzo[a,d]cyclo-hepten-5, 10-imine maleate (MK-801) impaired performance both on the place learn- ing version of the Morris (1981) swim task and on taste potentiation of an acquired odor aversion task.

We think this claim is unwarranted. Robinson et al.'s (1989) conclusion presupposes that MK-801 produced its behavioral effect through its actions at the NMDA receptor. There is evidence that MK-801 can block the induction of NMDA-dependent LTP in vivo (Abraham \& Mason, 1988). It should be noted, however, that a near lethal dose $(1.0-\mathrm{mg} / \mathrm{kg})$ was required to produce the effect, and such a dose would render the animal untestable on most behavioral tasks. Even in this case, however, Abraham and Mason reported that the MK-801 blockade of LTP did not emerge until 150 min after administration of MK-801, and Robinson et al. (1989) had completed the behavioral testing within 45 min of drug administration. Moreover, the LTP blockade that Abraham and Mason (1988) reported was associated with both a deterioration of the population spike evoked by single pulse stimulation prior to tetanization and a smaller response evoked during tetanization. That is, MK-801 at this high dose interferes with neural transmission in the hippocampus, and perhaps elsewhere, evoked by both high- and low-frequency stimulation. It has also been reported that MK-801 does not block the induction of LTP in vivo, even when animals receive doses so large that they cannot be tested in the swim task (Halliwell \& Morris, 1987).

In the absence of evidence that MK-801 blocked the induction of NMDA-dependent LTP, Robinson et al.'s (1989) claim that their behavioral impairments induced by this drug support the NMDA memory mechanism hypothesis is gratuitous. This criticism alone is sufficient reason for dismissing results obtained with MK-801 as providing support for the NMDA-dependent LTP memory hypothesis. In addition, however, it is well documented that MK-801 can produce significant sensory and motor impairments. For example, Mondadori et al. (1989) recently assessed the effects of MK-801 on place learning in the Morris swim task and emphasized the fact that MK-801 produces substantial sensorimotor impairments: "Behavioral abnormalities (hyperactivity, ataxia) were noted at the doses at which impaired learning occurred" (Mondadori et al., 1989, p. 455).

Finally, there is evidence that animals treated with MK-801 are capable of place learning in spite of the sensorimotor effects of the drug (Halliwell \& Morris, 1987). In Halliwell and Morris's study, the MK-801treated animals displayed impaired performance (longer escape latencies) during acquisition, but when subsequently tested while the drug was no longer present, they performed as well as the control animals.

\section{Other Forms of LTP}

Our primary purpose in this paper has been to develop an alternative interpretation of the existing data relating NMDA-receptor-dependent LTP to learning and memory. Thus, it is important to recognize that we are not arguing against the general hypothesis that learning and 
memory are dependent on altered synaptic strengths produced by experience. We are arguing that changes in synaptic strengths that depend on the activation of NMDA receptors are unlikely to be one of the mechanisms required for hippocampally mediated learning and memory in the adult mammalian brain. In fact, not all forms of long-term potentiation involve the NMDA-type receptor. For example, the induction of LTP in the mossy fibers of the hippocampus is not blocked by NMDA-receptor antagonists (Harris \& Cotman, 1986). Moreover, behavioral training on a variety of tasks is accompanied by changes in synaptic efficacy (Laroche \& Bloch, 1982; Sharp, McNaughton, \& Barnes, 1985; Thompson, Berger, \& Madden, 1983) that have not been shown to depend on the activation of NMDA receptors.

It is interesting to note, however, that one can make the argument that opioid-dependent LTP found in both the lateral perforant pathway (Bramham, Errington, \& Bliss, 1988) and area CA3 of the hippocampus (Martin, 1983 ) is also not involved in learning and memory tasks that are hippocampal-dependent. Opiate antagonists such as naloxone that are known to block the induction of this form of LTP have in fact been reported to enhance performance on a number of learning tasks that depend on hippocampally mediated processes (Decker, IntroiniCollison, \& McGaugh, 1989; Flood, Cherkin, \& Morley, 1987; Gallagher, 1985; Gallagher, Bostock, \& King, 1985; Izquierdo, 1979). Most recently, for example, Decker et al. (1989) reported that injections of naloxone given prior to training enhanced acquisition of the place learning version of the Morris swim task.

\section{CONCLUSIONS}

The NMDA-receptor blockade strategy has not provided support for the hypothesis that NMDA-receptordependent synaptic changes are crucial for some forms of learning and memory. No one has convincingly ruled out sensorimotor interpretations of the behavioral impairments that accompany the administration of AP5. The behavioral impairments produced by AP5 may be due to its anxiolytic, ataxic, or muscle relaxant properties. We also conclude that impaired performance produced by the noncompetitive agonist MK-801 cannot support the hypothesis, because MK-801 has no measurable effect on the induction of LTP at doses that permit the animal to behave.

The novel conclusion suggested by our review, however, is that several findings argue against the hypothesis that NMDA-receptor-dependent synaptic changes are involved in learning and memory. Specifically, AP5 blocks the induction of high-frequency stimulation-induced LTP in vivo in parts of the hippocampus known to be critical for hippocampally mediated learning; and in spite of this blockade (and the sensorimotor and motivational impairments that may accompany its administration), animals display learning both in situations that are thought to be dependent on the normal functioning of the hippocampus and in situations that do not depend on normal hippocampal function.

If it is indeed the case that animals learn and remember when NMDA-receptor-dependent synaptic changes are blocked, one would have to concede that these synaptic changes are unlikely to be critically involved in learning and memory. One might argue, however, that the learning observed by Staubli et al. (1989) and Morris et al. (1986) resulted because AP5 only partially blocked NMDA-dependent LTP in the hippocampus. It is certainly possible that not all NMDA receptors were blocked. ${ }^{3} \mathrm{Un}$ fortunately, however, if one is going to argue that the case against NMDA-receptor-dependent LTP as a memory mechanism can be made only if one has evidence that every NMDA receptor in the hippocampus was simultaneously blocked during the learning episode, then the hypothesis will be difficult to evaluate experimentally and may be impossible to refute.

\section{REFERENCES}

Abraham, W. C., Mason, S. E. (1988). Effects of the NMDA receptor/channel antagonists CPP and MK801 on hippocampal field potentials and long-term potentiation in anesthetized rats. Brain Research, 462, 40-46.

BenNeT, D. A., \& AMRICH, C. L. (1986). 2-amino-7-phosphonheptanoic acid (AP7) produces discriminative stimuli and anticonflict effects similar to diazepam. Life Sciences, 39, 2455-2461

BuIss, T. V. P., \& GARDNer-Medwin, A. R. (1973). Long-lasting potentiation of synaptic transmission in the dentate area of the unanaesthe tized rabbit following stimulation of the perforant path. Journal of Physiology, 232, 357-374.

BLIss, T. V. P., Lomo, T. (1973). Long-lasting potentiation of synaptic transmission in the dentate area of the anesthetized rabbit following stimulation of the perforant path. Journal of Physiology, 232, 331-356.

Bramham, C. R., Errington, M. L., \& Bliss, T. V. P. (1988). Naloxone blocks the induction of long-term potentiation in the lateral but not the medial perforant pathway in the anesthetized rat. Brain Research, 449, 352-356.

Brown, T. H., Chapman, P. F., Kairiss, E. W., \& Keenan, C. L. (1988). Long-term potentiation. Science, 242, 724-728.

Cahusac, P. M. B., Evans, R. H., Hill, R. G., Rodriguez, R. E., \& SMITH, D. A. S. (1984). The behavioral effects of an N-methyl-Daspartate receptor antagonist following application to the lumbar spinal cord of conscious rats. Neuropharmacology, 23, 719-724.

Clineschmidt, B. V., MArtin, G. E., \& Bunting, P. R. (1982). Anticonvulsant activity of $(+)-5$-methyl-10,11-dihydro-5Hdibenzo[a,d]cyclo-hepten-5,10-imine maleate (MK-801), a substance with potent anticonvulsant, central sympathomimetic, and apparent anxiolytic properties. Drug Development Research, 2, 123-134.

Collingridge, G. L., Kehl, S. J., \& Mclennan, H. (1983). Excitatory amino acids in synaptic transmission in the Schaffer-commissural pathway of the rat hippocampus. Journal of Physiology, 334, 33-46.

Compton, R. P., Kreiter, P. A., Smith, R. G., Harken, R. D., \& Monahan, J. B. (1988). Determination of the pharmacokinetics of 2-amino-7-phosphonoheptanoate in rat plasma and cerebrospinal fluid. Neuroscience Letters, 84, 339-344.

Decker, M. W., InTroini-Collison, I. B., \& MCGaugh, J. L. (1989). Effects of naloxone on Morris water maze learning in the rat: Enhanced acquisition with pretraining but not posttraining administration. Psychobiology, 17, 270-275.

Flood, J. F., Cherkin, A., \& Morley, J. E. (1987). Antagonism of endogenous opioids modulates memory processing. Brain Research, 422, 218-234. 
Gallagher, M. (1985). Re-viewing modulation of learning and memory. In N. Weinberger, J. McGaugh, \& G. Lynch (Eds.), Memory systems of the brain: Animal and human processes (pp. 311-334). New York: Guilford.

Gallagher, M., Bostock, E., \& KING, R. (1985). Effects of opiate antagonists on spatial memory in young and aged rats. Behavioral \& Neural Biology, 44, 374-385.

Halliwell, R. F., \& MoRRIS, R. G. M. (1987). Anticonvulsant doses of MK-801 fail to block long-term potentiation in vivo, or impair spatial memory in the rat. Neuroscience Letters Supplement, 29, 599.

HARRIS, E. W., \& Cotman, C. W. (1986). Long-term potentiation of guinea pig mossy fiber responses is not blocked by $\mathrm{N}$-methyl-Daspartate antagonists. Neuroscience Letters, 70, 132-137.

Harris, E. W., Evans, R. H., Francis, A. A., Jones, A. W., SMITH, D. A. S., \& WatKINS, J. C. (1982). The effects of a series of $w$-phosphonic alpha-carboxylic amino acids on electrically evoked and excitant amino acid-induced responses in isolated spinal cord preparations. British Journal of Pharmacology, 75, 65-75.

Harris, E. W., Ganong, A. H., \& Cotman, C. W. (1984). Longterm potentiation in the hippocampus involves activation of $\mathrm{N}$-methylD-aspartate receptors. Brain Research, 323, 132-137.

Headley, P. M., Parsons, C. G., \& West, D. C. (1987). The role of $\mathrm{N}$-methylaspartate receptors in mediating responses of rat and cat spinal neurones to defined sensory stimuli. Journal of Physiology, $385,169-188$.

IZQUIERDO, I. (1979). Effect of naloxone and morphine on various forms of memory in the rat: Possible role of endogenous opiate mechanisms in memory consolidation. Psychopharmacology, 66, 199-203.

Koeck, W., Woods, J. H., \& Ornstein, P. (1986). Phencyclidinelike behavioral effects in pigeons induced by systemic administration of the excitatory amino acid antagonist, 2-amino-5-phosphonovalerate. Life Sciences, 39, 973-978.

LAROCHE, S., \& BLOCH, V. (1982). Conditioning of hippocampal cells and long-term potentiation: An approach to mechanisms of posttrial memory formation. In C. A. Marsan and H. Matthies (Eds.), Neuronal plasticity and memory formation (pp. 575-587). New York: Raven.

Leung, L. S., \& Desborough, K. A. (1988). APV, an N-methyl-Daspartate receptor antagonist, blocks the hippocampal theta rhythm in behaving rats. Brain Research, 463, 148-152.

MaCKINTOSH, N. J. (1974). The psychology of animal learning. New York: Academic Press.

MARTIN, M. R. (1983). Naloxone and long-term potentiation of hippocampal CA3 field potentials in vitro. Neuropeptides, 4, 45-50.

McCrimmon, D. R., Smith, J. C., \& Feldman, J. L. (1989). Involvement of excitatory amino acids in neurotransmission of inspiratory drive to spinal respiratory motoneurons. Journal of Neuroscience, 9 , 1910-1921.

MCNaughton, N., \& MorRis, R. G. M. (1987). Chlordiazepoxide, an anxiolytic benzodiazepine, impairs place navigation in rats. $B e-$ havioral Brain Research, 24, 39-46.

Mondador, C., Weiskrantz, L., Buerki, H., Petschke, F., \& FAGG, G. E. (1989). NMDA receptor antagonists can enhance or impair learning performance in animals. Experimental Brain Research, 75, 449.456

MorRIs, R. G. M. (1981). Spatial localization does not require the presence of local cues. Learning \& Motivation, 12, 239-260.

Morris, R. G. M., ANDERson, E., LYNCH, G. S., \& BAUDRY, M. (1986). Selective impairment of learning and blockade of long-term potentiation by an $N$-methyl-D-aspartate receptor antagonist, AP5. Nature, 319, 774-776.

Pouc, P. (1985). 2-amino-7-phosphonoheptanoic acid depresses gammamotoneurons and polysynaptic reflexes in the cat spinal cord. European Journal of Pharmacology, 117, 774-776.

Robinson, G. S., JR., Crooks, G. B., JR., Shinkman, P. G., \& GALLAGHER, M. (1989). Behavioral effects of MK-801 mimic deficits associated with hippocampal damage. Psychobiology, 17, 156-164.

SALT, T. E. (1986). Mediation of thalamic sensory input by both NMDA receptors and non-NMDA receptors. Nature, 322, 263-265.
Sharp, P. E., McNaughton, B. L., \& Barnes, C. A. (1985). Enhancement of hippocampal field potentials in rats exposed to a novel, complex environment. Brain Research, 339, 361-365.

Staubl, U., Thibault, O., Dilorenzo, M., \& LynCh, G. (1989). Antagonism of NMDA receptors impairs acquisition but not retention of olfactory memory. Behavioral Neuroscience, 103, 54-60.

Sutherland, R. J., \& Dyck, R. H. (1984). Place navigation by rats in a swimming pool. Canadian Journal of Psychology, 38, 322-347.

Sutherland, R. J., Whishaw, I. Q., \& KolB, B. (1983). A behavioural analysis of spatial localization following electrolytic, kainate- or cholchicine-induced damage to the hippocampal formation in the rat. Behavioural Brain Research, 7, 133-153.

TeYler, T. J., \& DiScenNa, P. (1987). Long-term potentiation. Annual Review of Neuroscience, 10, 131-161.

Thompson, R. F., Berger, T. W., \& Madden, J. (1983). Cellular processes of learning and memory in the mammalian CNS. Annual Review of Neuroscience, 6, 447-491.

Tricklebank, M. D., Singh, L., Oles, R. J., Wong, E. H. F., \& IVERSEN, S. D. (1987). A role for receptors of N-methyl-D-aspartic acid in the discriminative stimulus properties of phencyclidine. European Journal of Pharmacology, 141, 497-501

Turskı, L., Schwartz, M., Turski, W. A., Klockgether, T., SontaG, K. H., \& Collins, J. F. (1985). Muscle relaxant action of excitatory amino acid antagonists. Neuroscience Letters, 53, 321-326.

\section{NOTES}

1. Morris et al. (1986) found that the infusion of AP5 (intraventricular) blocked LTP on Days 5-12 after the chronic implantation of minipumps containing AP5. This period corresponds to that during which behavioral testing was carried out in the place learning experiment. In addition, AP5 treatment caused "a total blockade of the induction of LTP" by high-frequency electrical stimulation (Morris et al., 1986, p. 776). Although we have no reason to reject the claim that intraventricularly administered AP5 blocks the induction of NMDA-dependent LTP, it should be pointed out that in neither the Morris et al. (1986) nor the Staubli et al. (1989) study was LTP assessed in the animals that were used for behavioral testing.

2. To evaluate the effect of AP5 on place learning independently of its sensorimotor effects, Morris et al. (1986) could have included in their experiment animals that were treated with AP5 and trained with the platform randomly placed at a different position in the pool on each trial. When the platform is in a different spatial location on each trial, spatial information is of no value to the animal in solving the problem. Consequently, one can use the random platform condition to assess the degree to which the relatively long escape latencies of AP5-treated animals reflect learning about the spatial location of the platform as opposed to learning nonspatial aspects of the task such as climbing onto the platform and swimming the appropriate distance from the wall. If an animal trained with the platform in the same location on each trial cannot or does not learn about the spatial location of the platform, its escape latencies during training should not differ from those of animals trained with the platform in a random location on each trial.

If the escape latencies of animals treated with AP5 reflect a selective effect on place learning processes and are not due to the sensorimotor effect of the agent, then the escape latencies of AP5-treated animals trained with the platform always in a fixed location would not differ from the escape latencies of APS-treated animals trained with the platform randomly placed in a different quadrant on each trial. Morris (1981) demonstrated the usefulness of employing a random platform location condition as a control in his original demonstration of place learning in a swimming pool.

3. It seems likely, however, that an infusion of AP5 in doses sufficient to completely block high-frequency electrical stimulation-induced LTP should unequivocally impair learning and memory, unless the neuronal changes necessary for learning are not NMDA/LTP-dependent.

(Manuscript received August 8, 1989; revision accepted for publication June 5, 1990.) 